\title{
Anti-proteolytic capacity and bonding durability of proanthocyanidin-biomodified demineralized dentin matrix
}

\author{
Rui-Rui Liu ${ }^{1,2, *}$, Ming Fang ${ }^{1, *}$, Ling Zhang ${ }^{1}$, Cheng-Fang Tang ${ }^{1}$, Qi Dou ${ }^{1}$ and Ji-Hua Chen ${ }^{1}$
}

Our previous studies showed that biomodification of demineralized dentin collagen with proanthocyanidin (PA) for a clinically practical duration improves the mechanical properties of the dentin matrix and the immediate resin-dentin bond strength. The present study sought to evaluate the ability of PA biomodification to reduce collagenase-induced biodegradation of demineralized dentin matrix and dentin/adhesive interfaces in a clinically relevant manner. The effects of collagenolytic and gelatinolytic activity on PA-biomodified demineralized dentin matrix were analysed by hydroxyproline assay and gelatin zymography. Then, resin-/dentin-bonded specimens were prepared and challenged with bacterial collagenases. Dentin treated with $2 \%$ chlorhexidine and untreated dentin were used as a positive and negative control, respectively. Collagen biodegradation, the microtensile bond strengths of bonded specimens and the micromorphologies of the fractured interfaces were assessed. The results revealed that both collagenolytic and gelatinolytic activity on demineralized dentin were notably inhibited in the PA-biomodified groups, irrespective of PA concentration and biomodification duration. When challenged with exogenous collagenases, PA-biomodified bonded specimens exhibited significantly less biodegradation and maintained higher bond strengths than the untreated control. These results suggest that PA biomodification was effective at inhibiting proteolytic activity on demineralized dentin matrix and at stabilizing the adhesive/dentin interface against enzymatic degradation, is a new concept that has the potential to improve bonding durability.

International Journal of Oral Science (2014) 6, 168-174; doi:10.1038/ijos.2014.22; published 9 May 2014

Keywords: bonding durability; collagenolysis; crosslinking agents; dentin; proanthocyanidins

\section{INTRODUCTION}

There is common consensus that resin-dentin bonds created with contemporary hydrophilic dentin bonding systems deteriorate over time. ${ }^{1-2}$ Collagen disorganisation in the incompletely resin-infiltrated demineralized dentin matrix is one of the main degradation patterns found in unsuccessful adhesive restorations. ${ }^{3}$ Continuing efforts have focused on preservation of the integrity of the collagen matrix of the hybrid layer in an attempt to achieve durable dentin bonding. Considering the key role of matrix metalloproteinases (MMPs) in interfacial ageing over time, inhibiting the activity of host-derived MMPs and reducing the susceptibility of collagen matrices to MMP-induced degradation via bonding procedures may thus be a rational and effective approach for the improvement of bonding durability. $^{2}$

Many attempts have been made to apply MMP inhibitors to acidetched dentin prior to the application of adhesives. ${ }^{4-6}$ The only such inhibitor that has proved to be effective for reducing the degradation of resin-dentin bonds after in vivo ageing is chlorhexidine (CHX), a classic nonspecific MMP inhibitor. ${ }^{7}$ However, its MMP-inhibitory effect lasts only 9-12 months, and then weakens over time as CHX slowly leaches from the resin/dentin interface. ${ }^{8}$ Proanthocyanidins (PAs), a group of naturally occurring polyphenolic compounds, have the potential to control MMP-mediated diseases, such as periodontitis, by inhibiting both MMP production and activity. ${ }^{9}$ Epasinghe et al. ${ }^{10}$ demonstrated that PA can inactivate more than $90 \%$ of soluble recombinant MMP-2, -8 and -9 and approximately $70 \%-80 \%$ of cysteine cathepsin B and $\mathrm{K}$; these results are significantly better those derived from the use of $\mathrm{CHX}$.

Apart from being a potential non-selective MMP inhibitor, PA has gained much attention over the past few years because of its crosslinking capabilities, which are beneficial for enhancing demineralized dentin matrix and improving resin-dentin bond strength. Castellan et al. ${ }^{1-13}$ reported that PA pre-treatment increased the immediate elastic modulus of dentin matrix and was effective even after bacterial collagenase challenge or 1-year of storage in artificial saliva. Additionally, it provided enhanced immediate adhesion and long-term stabilisation to demineralized dentin after 1-year of ageing in water. Hechler et al. ${ }^{14}$ evaluated the long-term performance of PA application both as an additive to the adhesive and as a primer in an extra bonding step. They found that after 52 weeks' exposure to collagenase digestion, the

${ }^{1}$ State Key Laboratory of Military Stomatology, Department of Prosthodontics, School of Stomatology, The Fourth Military Medical University, Xi'an, China and ${ }^{2}$ Department of Prosthodontics, Stomatology Hospital of Xi'an Jiaotong University College of Medicine, Xi'an, China

*These authors contributed equally to this work.

Correspondence: Professor JH Chen, State Key Laboratory of Military Stomatology, Department of Prosthodontics, School of Stomatology, The Fourth Military Medical University, No.145 Changle Xi Road, Xi'an 710032, China

E-mail: jhchen@fmmu.edu.cn

Accepted 26 February 2014 
bond strength of the PA-primer group was significantly higher than that of the control, whereas no significant difference was found between the PA-adhesive group and the control. This observation strongly supports the use of PA as a primer. However, all of the above-mentioned studies had a common limitation: the PA priming time was over $10 \mathrm{~min}$, which is not clinically practical. An extra bonding step in PA biomodification for a clinically relevant duration improved the mechanical properties of demineralized dentin collagen ${ }^{15}$ as well as the immediate resin-dentin bond strength to dentin, ${ }^{16}$ and was effective at reducing the interfacial ageing caused by thermal cycling. ${ }^{17}$

The combined crosslinking and potential anti-collagenolytic effects of PA would be beneficial in preventing degradation of dentin collagen within the hybrid layer. Using a gravimetric collagenase digestion assay, Liu et al. ${ }^{18}$ indirectly showed that PA biomodification for clinically relevant time periods (as short as $10 \mathrm{~s}$ and $1 \mathrm{~min}$ ) can enhance dentinal collagen's resistance towards enzymatic challenge. Nevertheless, further studies are still needed to directly confirm the anti-proteolytic effect of PA applied to demineralized dentin matrix for clinically relevant time periods, and its role in improving the resistance of the bonding interface to enzymatic degradation. Therefore, the aims of the present study were to investigate the potential effect of PA on endogenous gelatinolytic activity on demineralized dentin matrices, as well as to determine the capability of PA biomodification to reduce collagen biodegradation in demineralized dentin matrices and adhesive/dentin interfaces on challenge with bacterial collagenases in a clinically relevant manner. The null hypothesis was that transient PA biomodification would have no effect on gelatinolytic activity on demineralized dentin and would not increase the resistance of the dentin matrix or the bonding interface to collagenolysis.

\section{MATERIALS AND METHODS}

Extracted intact human third molars were collected with patients' informed consent, under a protocol approved by the Ethics Committee Board of the Fourth Military Medical University. The teeth were cleaned of adhering soft tissue, stored in physiological saline at $4{ }^{\circ} \mathrm{C}$ and used within 2 weeks of extraction. PA biomodifiers were prepared by dissolving PA-rich $(\geqslant 95 \%)$ grape seed extract (Vitis vinifera L; Acetar Bio-Tech, Xi'an, China) into ethanol at 15\% and 10\%. CHX (2\%) (DaSheng Chemical Tech, Xi'an, China) was used for the pretreatment of positive controls, and untreated teeth were used as negative controls. The experimental design and procedures are summarized in Figure 1.

\section{Collagenolytic/gelatinolytic activity on PA-biomodified demineralized dentin matrix}

Collagenase-mediated biodegradation. The effect of PA biomodification on collagenolytic activity on demineralized dentin matrix was assessed by measuring the hydroxyproline (Hyp) released from demineralized dentin after enzymatic challenge. Collagenase solutions were prepared by dissolving collagenase type I and type II (from Clostridium histolyticum, $\geqslant 125$ collagen digestion units (CDU) per mg solid; Sigma-Aldrich, St Louis, MO, USA) in $0.2 \mathrm{mmol} \cdot \mathrm{L}^{-1}$ phosphate-buffered solution $(\mathrm{pH}=7.4)$ at a concentration of $0.2 \%(\mathrm{~m} / \mathrm{m})$. Completely demineralized dentin slabs of approximately $0.5 \mathrm{~mm} \times 1.7 \mathrm{~mm} \times 7.0 \mathrm{~mm}$ were prepared from the mid-coronal dentin of 40 mol. ${ }^{15}$ Forty slabs were randomly assigned to each group based on the treatment (pre-treated with $15 \%$ PA, $10 \%$ PA or $2 \%$ CHX for 60 s, 120 s or 0 s (without pretreatment)). Then, the slabs in each group were equally subdivided into two subgroups and exposed to $1 \mathrm{~mL}$ of either collagenase type I or type II solution at $37^{\circ} \mathrm{C}$, until the untreated specimens had completely disappeared, as judged by the naked eye. The collagenase solutions were changed every $24 \mathrm{~h}$ during the course of exposure. A $50 \mu \mathrm{L}$ aliquot of the supernatant was collected for each group and was subjected to Hyp content analysis (Hydroxyproline Assay Kit; SigmaAldrich, St Louis, MO, USA) at pre-arranged time points, following the manufacturer's instructions. The absorbance was measured at $560 \mathrm{~nm}$ using a spectrophotometer (Tecan Group, Männedorf, Switzerland). The Hyp content in each group was determined as the average of duplicate measurements. The amount of dissolved collagen from demineralized dentin slabs was expressed as micrograms of hydroxyproline per milligram of the dry mass of the demineralized dentin before incubation. ${ }^{19-20}$

Activity of host-derived gelatinases. Seven aliquots (1 g each) of demineralized dentin powder were obtained and subjected to the above-mentioned biomodification. Then, specimens in each group were incubated in $1 \mathrm{~mL}$ of Adper Single Bond 2 (3M ESPE, St. Paul, MN, USA) for $24 \mathrm{~h}$ at $4{ }^{\circ} \mathrm{C}$ in the dark, thoroughly rinsed with acetone and repeatedly centrifuged $\left(4000 \mathrm{~g}\right.$ for $10 \mathrm{~min}$ each) at $4{ }^{\circ} \mathrm{C}$. Enzyme extraction and zymographic analyses were carried out as previously described $^{4,21}$ to evaluate the effect of PA on the gelatinolytic activity of host-derived MMPs on dentin.

Briefly, the specimens were suspended in the extraction buffer ( $50 \mathrm{mmol} \cdot \mathrm{L}^{-1}$ Tris- $\mathrm{HCl}, \mathrm{pH}$ 6.0) to ultrasonically extract the enzyme proteins. The supernatants were collected by centrifugation. Then, proteins were precipitated at $4{ }^{\circ} \mathrm{C}$ by adding powdered ammonium sulphate, redissolved and further dialysed through a $30-\mathrm{kDa}$ membrane overnight. Total protein concentrations in demineralized dentin powder extracts were determined by Bradford assay (Beyotime Biology, Haimen, China). Dentin proteins were electrophoresed under non-reducing conditions on $7.5 \%$ sodium dodecyl sulfate (SDS)-polyacrylamide gels copolymerized with $2 \mathrm{~g} \cdot \mathrm{L}^{-1}$ gelatin (GMS30071.1; Gemend, Shanghai, China). Gels were stained in 0.2\% Coomassie Brilliant Blue R-250 and destained. Wet gelatine zymograms were scanned using an EagleEye II imaging system (Stratagene, Santa Clara, CA, USA).

\section{Resistance of resin-dentin bonds to collagenase-mediated degradation}

Microtensile testing. Bond strength testing is the gold standard for the assessment of bonding durability. Changes in the microtensile bond strength ( $\mu \mathrm{TBS}$ ) after enzymatic challenge were used as an indirect measure of collagenase-induced biodegradation of resin-dentin bonds.

The occlusal surfaces of 105 teeth were ground flat to expose the middle coronal dentin and abraded to create a uniform smear layer under water cooling. Each tooth was further cut perpendicularly to the exposed dentin surface into four blocks. All blocks were randomly assigned to seven groups and pre-treated as mentioned above. The bonded specimens were prepared using Single bond 2 as previously described ${ }^{20}$ and sectioned perpendicularly to the bonding interface into beams with a rectangular dimension of $0.8 \mathrm{~mm} \times 0.8 \mathrm{~mm} \times 8.0 \mathrm{~mm}$, using a slowspeed diamond wafering blade under constant water irrigation.

All of the beams were stored in distilled water at $37{ }^{\circ} \mathrm{C}$ for $24 \mathrm{~h}$. Then, the beams in each group were equally subdivided into four subgroups and exposed to the aforementioned collagenase type I or type II solution for various durations ( $120 \mathrm{~h}$ or $24 \mathrm{~h}, 40$ beams for each subgroup). The collagenase solution was changed every $24 \mathrm{~h}$ during the experiment. After exposure, the specimens were rinsed before the $\mu$ TBS testing was performed in a universal testing machine (EZ-test; Shimadzu, Tokyo, Japan), at a crosshead speed of $0.5 \mathrm{~mm} \cdot \mathrm{min}^{-1}$. The means and standard deviations of $\mu$ TBS were calculated as previously described. ${ }^{22}$ 


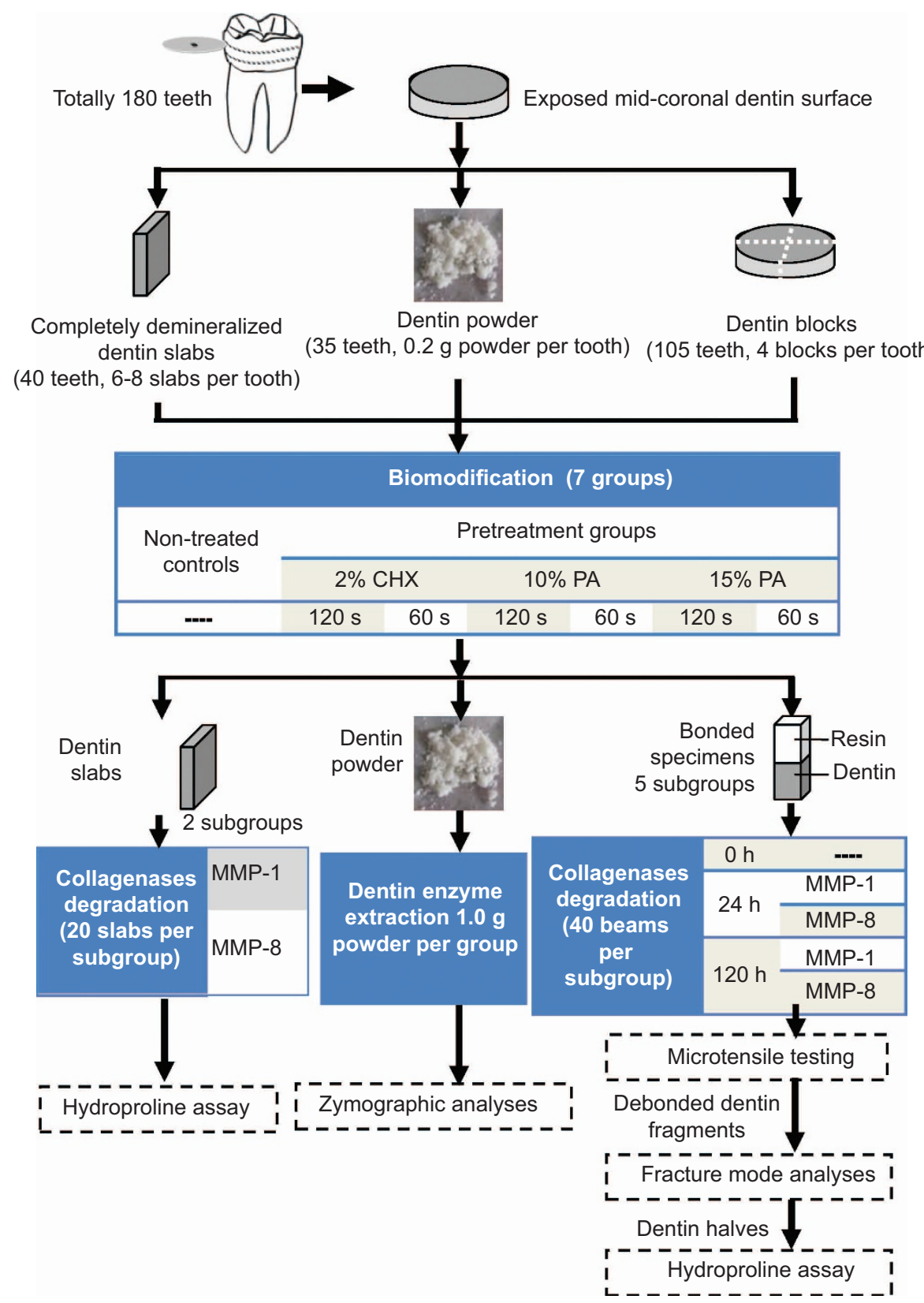

Figure 1 Schematic illustration of experimental design and procedures. $\mathrm{CHX}$, chlorhexidine; PA, proanthocyanidin.

Fracture mode analyses. All of the dentin fragments that had debonded from the specimens after $\mu$ TBS testing were collected to determine the fracture mode under a stereomicroscope (MLC-150; Motic, San Antonio, TX, USA) as previously described. ${ }^{16}$ These fragments were classified as resulting from adhesive failure, mixed failure or cohesive failure of the resin or the dentin. Two representative fractured beams from each group with a $\mu$ TBS close to the mean bond strength of that group were fixed, dried and gold sputter-coated for micromorphological evaluation under a field emission scanning electron microscope (S-4800; Hitachi, Tokyo, Japan).

Amount of collagen biodegradation. The amount of dissolved collagen from the resin-dentin bonding interface following collagenase digestion was measured as another, indirect, index of collagenaseinduced hydrolysis in dentin collagen matrices by using the Hyp assay. This assay was performed as previously described with minor modifications. ${ }^{20}$ After fracture mode analyses, dentin halves within $1 \mathrm{~mm}$ of the fractured surface were collected (specimens with cohesive failure in dentin were excluded because their dentin halves were not involved with the hybrid layer), pulverized in liquid nitrogen and then incubated in distilled water for $24 \mathrm{~h}\left(100 \mathrm{mg} \cdot \mathrm{mL}^{-1}\right)$ at $37{ }^{\circ} \mathrm{C}$ with gentle shaking. All mixtures were separated by centrifugation $(\times 1000 g, 5 \mathrm{~min})$ at $4{ }^{\circ} \mathrm{C}$. The supernatants were subjected to amino-acid analyses to determine the amount of dissolved collagen from the resin/dentin bonding interface as mentioned above.

\section{Statistical analysis}

Because the normality and homoscedasticity assumptions of the data were valid, a General Linear Model SPSS 13.0 program (SPSS, Chicago, IL, USA) for analysis of variance (ANOVA) (biomodification 
duration $v s$. exposure time to collagenase solution) was conducted to test the significance of variations in (i) biodegradation of demineralized dentin and (ii) $\mu$ TBS values and collagen biodegradation in bonding surfaces among groups challenged with collagenases. Multiple comparisons between groups were evaluated by Tukey's post hoc test. Differences were deemed significant at $P<0.05$.

\section{RESULTS}

Collagenolytic/gelatinolytic activity on PA-biomodified demineralized dentin matrix

Inhibitory effect on collagenase-mediated biodegradation. As observed with the naked eye, the untreated demineralized dentin slabs completely disappeared in collagenase type I or type II solution after $72 \mathrm{~h}$ or $60 \mathrm{~h}$ of exposure, respectively. In contrast, PA-biomodified specimens remained mostly intact, and defects could be seen in CHX-pre-treated specimens. Significantly greater collagen biodegradation was observed in the untreated control than in the other groups $(P<0.05)$ (Figure 2). All PA groups presented significantly lower collagen biodegradation than did the CHX groups at the same time point $(P<0.05)$. The lowest biodegradation was in the $15 \%$ PA-120 s group.

Inactivation of host-derived gelatinases. Multiple forms of gelatinases were detected in demineralized dentin powder after Single Bond 2 application without biomodification (Figure 3), including an intense band at $66 \mathrm{kDa}$ identified as the active form of MMP-2, a $72 \mathrm{kDa}$ band identified as proMMP-2 and 130 and $92 \mathrm{kDa}$ bands identified as the latent and activated MMP-9 forms. In addition, other minor gelatinolytic bands with lower molecular weights were fainter but detectable. Complete inhibition of all forms of MMP-2 and MMP9 in dentin matrices was exhibited in PA-biomodified groups.

\section{Resistance of resin-dentin bonds to collagenase-mediated} degradation

The $\mu$ TBS values of the PA groups were not significantly reduced after challenge with collagenase type I for $24 \mathrm{~h}$, irrespective of PA concentration and biomodification duration $(P>0.05)$. By contrast, the $\mu$ TBS values of the untreated control and $\mathrm{CHX}$ groups decreased
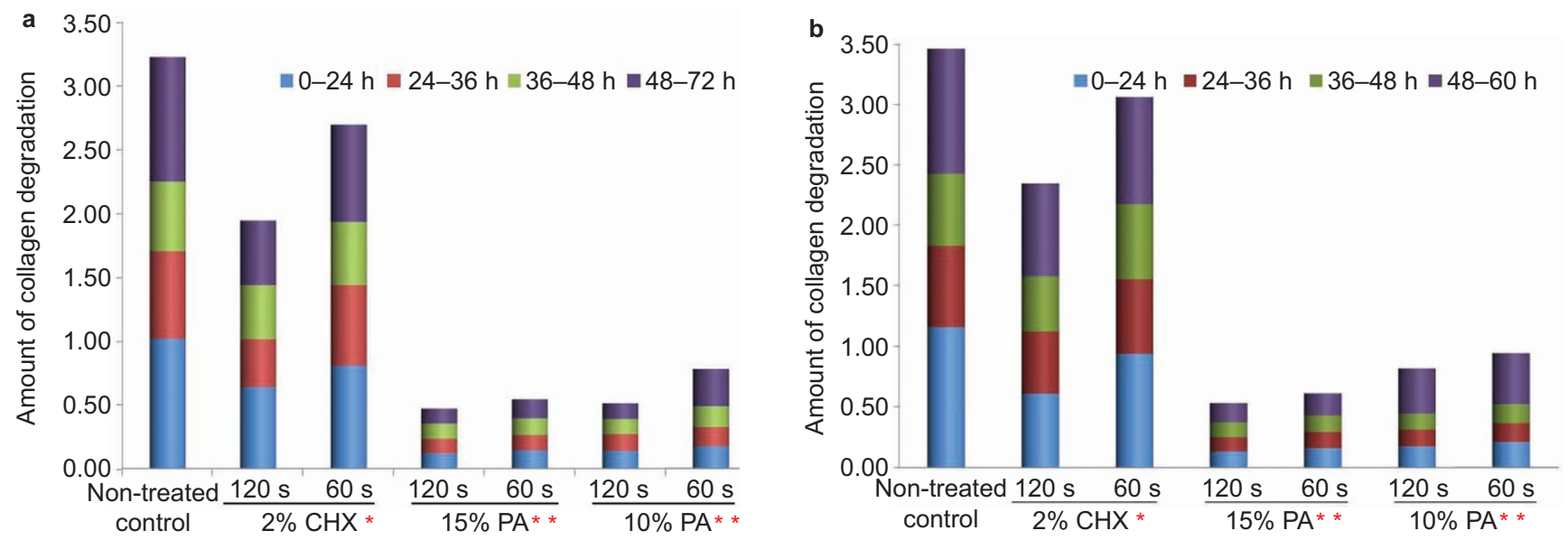

Figure 2 The amount of collagen biodegradation of demineralized dentin after challenge with collagenase. (a) Collagenase type I; (b) Collagenase type II. Amount of collagen degradation in $\times 10^{-4}, \mathrm{~m} / \mathrm{m}$. *Significantly lower collagen biodegradation was observed in the $2 \% \mathrm{CHX}$ group than the untreated group at the same time point $(P<0.05)$. **PA preconditioning significantly reduced the collagen biodegradation compared with the other groups, regardless of PA concentration and treatment duration $(P<0.01)$. CHX, chlorhexidine; PA, proanthocyanidin.

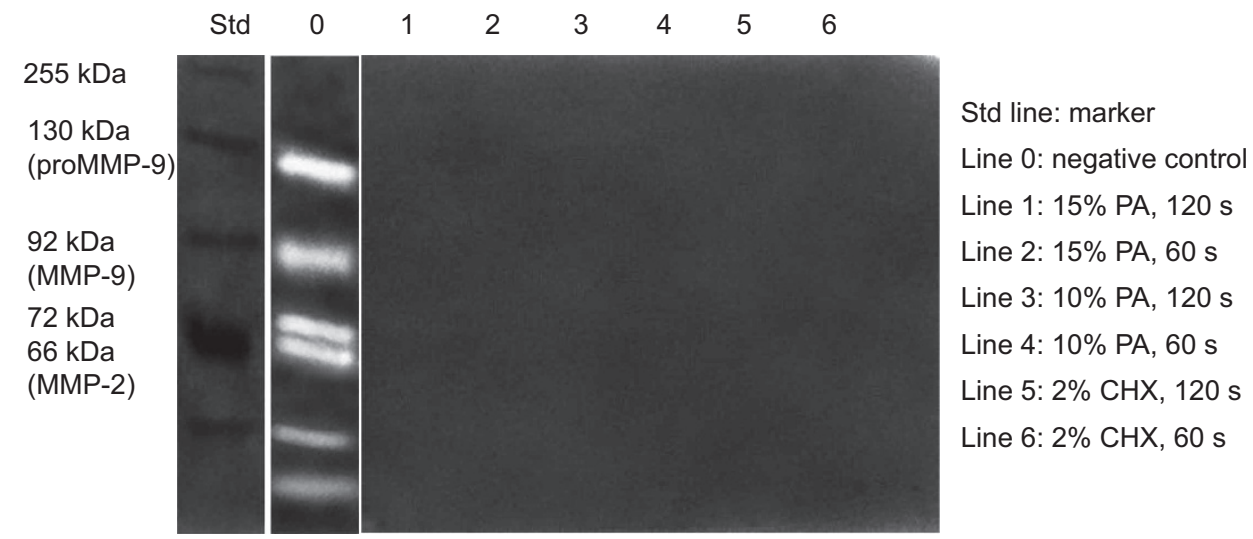

Figure 3 Gelatin zymography of MMPs from dentin extracts with or without PA pre-treatment. The relative molecular masses of MMP-2 and MMP-9, expressed in $\mathrm{kDa}$, are presented in Std lane. After pre-treatment, gelatinolytic bands produced by MMP-2 and MMP-9 were visible only in the untreated group. CHX, chlorhexidine; MMP, matrix metalloproteinase; PA, proanthocyanidin. 
Table $1 \mu$ TBS of bonded specimens with or without PA pre-treatment after collagenase challenge

\begin{tabular}{|c|c|c|c|c|c|c|c|c|}
\hline \multirow[b]{3}{*}{ Exposure time } & \multirow[b]{3}{*}{ Collagenase } & \multirow{3}{*}{$\begin{array}{l}\text { Non-treated } \\
\text { controls }\end{array}$} & \multicolumn{6}{|c|}{ Pre-treatment groups } \\
\hline & & & \multicolumn{2}{|c|}{$2 \% \mathrm{CHX}$} & \multicolumn{2}{|c|}{$10 \% \mathrm{PA}$} & \multicolumn{2}{|c|}{$15 \% \mathrm{PA}$} \\
\hline & & & $120 \mathrm{~s}$ & $60 \mathrm{~s}$ & $120 \mathrm{~s}$ & $60 \mathrm{~s}$ & $120 \mathrm{~s}$ & $60 \mathrm{~s}$ \\
\hline \multirow[t]{2}{*}{$24 \mathrm{~h}$} & Type I & $22.45^{\mathrm{a}, \mathrm{B}}(5.68)$ & $25.71^{\mathrm{a}, \mathrm{B}}(5.19)$ & $23.99^{\mathrm{a}, \mathrm{B}}(5.58)$ & $29.04^{\mathrm{b}, \mathrm{A}}(6.44)$ & $26.13^{\mathrm{a}, \mathrm{A}}(4.81)$ & $32.01^{\mathrm{b}, \mathrm{A}}(6.10)$ & $29.67^{\mathrm{b}, \mathrm{A}}(5.63)$ \\
\hline & Type II & $16.28^{\mathrm{a}, \mathrm{C}}(5.16)$ & $22.78^{\mathrm{b}, \mathrm{C}}(4.83)$ & $20.29^{\mathrm{b}, \mathrm{C}}(4.91)$ & $23.48^{\mathrm{b}, \mathrm{B}}(5.41)$ & $21.75^{\mathrm{b}, \mathrm{B}}(5.36)$ & $26.50^{c, B}(5.93)$ & $23.96^{\mathrm{b}, \mathrm{B}}(6.58)$ \\
\hline $120 \mathrm{~h}$ & Type I & $13.89^{\mathrm{a}, \mathrm{C}}(3.05)$ & $20.17^{\mathrm{b}, \mathrm{C}}(5.19)$ & $17.09^{\mathrm{a}, \mathrm{C}}(4.85)$ & $21.63^{\mathrm{b}, \mathrm{B}}(5.96)$ & $19.26^{\mathrm{b}, \mathrm{B}}(4.51)$ & $26.52^{c, B}(4.61)$ & $24.06^{\mathrm{c}, \mathrm{B}}(5.01)$ \\
\hline
\end{tabular}

CHX, chlorhexidine; PA, proanthocyanidin; $\mu \mathrm{TBS}$, microtensile bond strength.

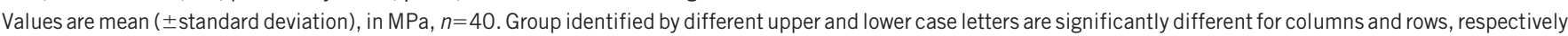
$(P<0.05)$.
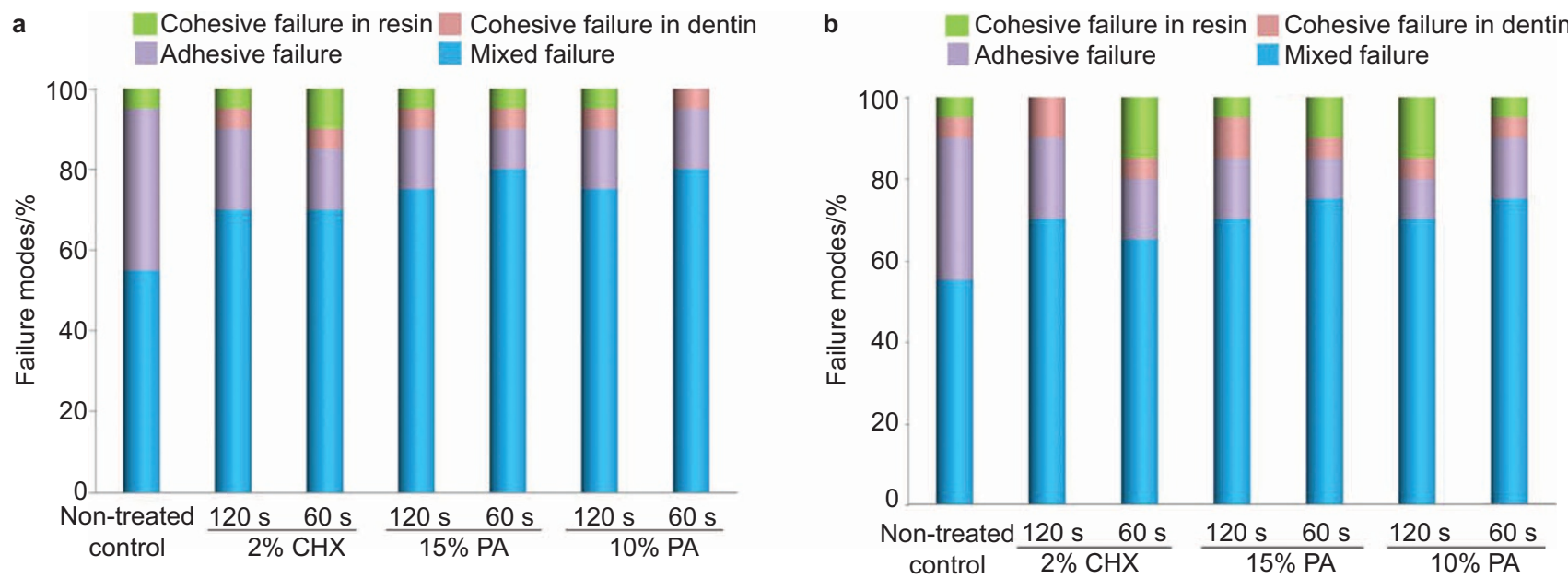

Figure 4 Comparison of the failure modes in different groups after exposed to collagenase type I or type II solution for $120 \mathrm{~h}$. (a) Collagenase type I; (b) Collagenase type II.

significantly $(P<0.05)$ (Table 1$)$. The $\mu$ TBS advantage of PA groups over the untreated control persisted even after $120 \mathrm{~h}$ exposure $(P<0.05)$, although the values decreased to some extent. The highest $\mu$ TBS value was exhibited by the $15 \%$ PA-120 s group. No significant differences were observed between the $10 \%$ PA (120 or $60 \mathrm{~s}$ ) groups and the CHX-120 s group. Exposure to collagenase type II for $24 \mathrm{~h}$ significantly reduced the $\mu$ TBS of all groups. Prolonging the exposure time to $120 \mathrm{~h}$ resulted in a notably decreased $\mu$ TBS for the untreated control $(P<0.05)$, whereas no significant changes occurred in the biomodified groups $(P>0.05)$.

Fracture mode analyses revealed that biomodified specimens exhibited a high percentage of mixed failures (Figure 4). Fractures mostly happened at the top of the hybrid layer, with numerous resin tags tightly filling the dentinal tubules, and intertubular dentin was partially sealed by resin (Figure $5 \mathrm{a}-5 \mathrm{c}$ ). Adhesive failures accounted for a large percentage of failures except for the mixed failures in the untreated control group, mostly at the bottom of the hybrid layer (Figure 5d).

Biomodified groups presented significantly less collagen biodegradation than the untreated control $(P<0.05)$ (Table 2$)$. No significant differences were found in collagen biodegradation among groups pre-treated for $120 \mathrm{~s}(P>0.05)$. Among those pre-treated for
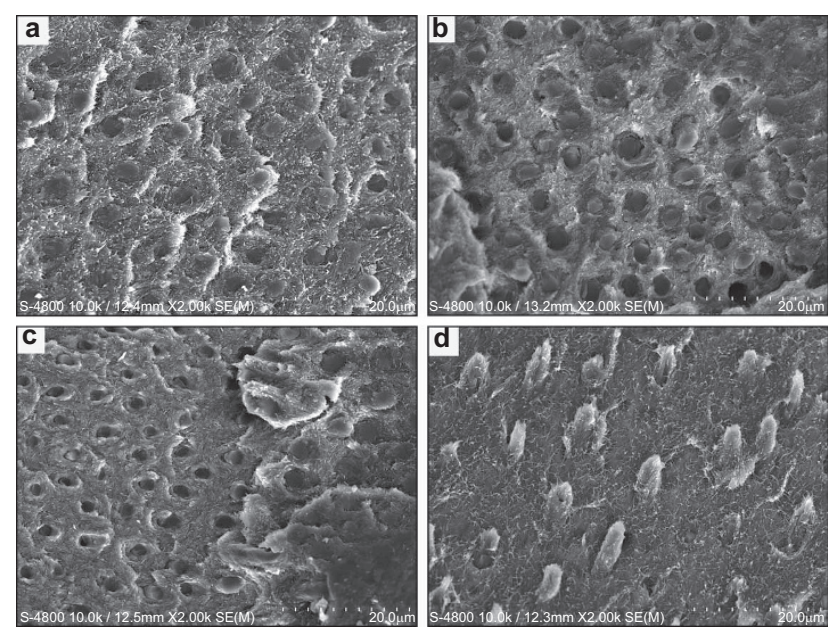

Figure 5 Representative FESEM micrographs of fractured dentin surfaces. (a) $15 \%$ PA-120 s group failed at the top of the hybrid layer; (b) Mixed failure happened at the top of hybrid layer was the most prevalent fracture mode in $10 \% \mathrm{PA}$ 120 s group; (c) $2 \% \mathrm{CHX}-120$ s group presented adhesive failure; (d) Non-treated control group fractured at the bottom of the hybrid layer. FESEM, field emission scanning electron microscope; PA, proanthocyanidin; $\mathrm{CHX}$, chlorhexidine. 
Table 2 Collagen biodegradation of bonded specimens with or without PA pre-treatment after collagenase challenge

\begin{tabular}{|c|c|c|c|c|c|c|c|c|}
\hline \multirow[b]{3}{*}{ Exposure time } & \multirow[b]{3}{*}{ Collagenase } & \multirow{3}{*}{$\begin{array}{c}\text { Non-treated } \\
\text { controls }\end{array}$} & \multicolumn{6}{|c|}{ Pre-treatment groups } \\
\hline & & & \multicolumn{2}{|c|}{$2 \% \mathrm{CHX}$} & \multicolumn{2}{|c|}{$10 \% \mathrm{PA}$} & \multicolumn{2}{|c|}{$15 \% \mathrm{PA}$} \\
\hline & & & $120 \mathrm{~s}$ & $60 \mathrm{~s}$ & $120 \mathrm{~s}$ & $60 \mathrm{~s}$ & $120 \mathrm{~s}$ & $60 \mathrm{~s}$ \\
\hline & Type II & $0.229^{a, c}(0.010)$ & $0.096^{\mathrm{b}, \mathrm{B}}(0.023)$ & $0.137^{\mathrm{b}, \mathrm{c}}(0.002)$ & $0.091^{\mathrm{b}, \mathrm{B}}(0.050)$ & $0.118^{\mathrm{b}, \mathrm{c}}(0.016)$ & $0.082^{b, B}(0.005)$ & $0.083^{\mathrm{b}, \mathrm{B}}(0.003)$ \\
\hline \multirow[t]{2}{*}{$120 \mathrm{~h}$} & Type I & $0.166^{\mathrm{a}, \mathrm{B}}(0.012)$ & $0.096^{\mathrm{b}, \mathrm{B}}(0.016)$ & $0.104^{c, B}(0.008)$ & $0.074^{\mathrm{b}, \mathrm{B}}(0.016)$ & $0.099^{\mathrm{b}, \mathrm{B}}(0.013)$ & $0.069^{b, B}(0.003)$ & $0.095^{\mathrm{b}, \mathrm{B}}(0.001)$ \\
\hline & Type II & $0.256^{\mathrm{a}, \mathrm{D}}(0.020)$ & $0.169^{b, c}(0.002)$ & $0.217^{\mathrm{c}, \mathrm{D}}(0.016)$ & $0.154^{\mathrm{b}, \mathrm{c}}(0.022)$ & $0.187^{\mathrm{b}, \mathrm{D}}(0.016)$ & $0.145^{\mathrm{b}, \mathrm{c}}(0.009)$ & $0.173^{\mathrm{b}, \mathrm{C}}(0.009)$ \\
\hline
\end{tabular}

CHX, chlorhexidine; PA, proanthocyanidin.

Values are mean ( \pm standard deviation), in $\times 10^{-4}, \mathrm{~m} / \mathrm{m}$. Group identified by different upper and lower case letters are significantly different for columns and rows, respectively $(P<0.05)$.

$60 \mathrm{~s}$, the 15\% PA group presented the least collagen degradation, followed by the $10 \% \mathrm{PA}$ and $2 \% \mathrm{CHX}$ groups, regardless of the type of collagenase and the exposure time $(24 \mathrm{~h}$ or $120 \mathrm{~h})(P<0.05)$.

\section{DISCUSSION}

Several MMPs, such as MMP-1, -2, -8 and -9, are secreted as proenzymes in the human dentin matrix and in saliva. These enzymes have been suggested to be activated in the low-pH environment of the mouth and to be responsible for the progressive breakdown of collagen matrices in resin-sparse regions at the bottom of bonding interfaces. $^{21,23}$ Two mechanisms have been proposed to explain the degradation of collagen fibrils: namely, collagenolysis and gelatinolysis. Collagenases (e.g., MMP-1, -8) and gelatinase A (MMP-2) can cleave collagens into 1/4- and 3/4-fragments at the Gly-Leu/Ile peptide bond, where the collagen peptide structure determines both the specific cleavage and the binding sites for MMPs. ${ }^{24}$ The fragments then denature at body temperature and can be further degraded by gelatinases (e.g., MMP-2, -9) and other nonspecific tissue proteinases. ${ }^{25}$ As MMP-2 and MMP-9 are distributed widely in mature human coronal dentin, ${ }^{26}$ we directly evaluated the activity of these host-derived gelatinases on PA-biomodified dentin matrix by gelatin zymography. We indirectly assessed the collagenolytic activity of bacterial collagenases on PA-biomodified demineralized dentin matrix by measuring the release of Hyp into hydrolysates of media because only small amounts of endogenous collagenases (e.g., MMP-1, -8) are present in dentin matrix. The results revealed that transient PA biomodification was effective at inhibiting proteolytic activity and markedly improved the resistance of bonding interfaces to enzymatic degradation. The null hypothesis was consequently rejected.

CHX, a classic non-selective MMP inhibitor, was used for the pretreatment of positive controls in this study because the application of $2 \% \mathrm{CHX}$ to the demineralized dentin surface is the only procedure that has been proven to be clinically effective against bond degradation. ${ }^{7,27}$ Importantly, the $\mu$ TBS of the PA groups was significantly higher than that of the CHX groups after collagenase exposure, yet the extent of collagen biodegradation was similar (Table 2). This finding may be ascribed to the considerable proportion of mineralized dentin involved in the specimens (dentin halves within $1 \mathrm{~mm}$ of the fractured surface). This may have reduced the differences between the collagenase-mediated biodegradation of PA groups and that of $\mathrm{CHX}$ groups, leading to no significant difference (Table 2). In light of this finding, the results of the collagenase-mediated biodegradation of completely demineralized dentin slabs exposed to a high concentration of collagenase solution (the section on 'Collagenase-mediated biodegradation') may provide a more realistic assessment of the clinical situation and can be used as a reference for the long-term assessment of dentin biomodification on resistance to collagenase challenge.
Figure 2 shows that PA exhibited significantly higher protease inactivation potential than $\mathrm{CHX}$ at the same exposure duration. This result may be ascribed to the use of a higher concentration of PA than of CHX because PA has better biocompatibility than CHX. Additionally, the distinct modes of action of PA and CHX in stabilising collagen could explain the superiority of PA. CHX is capable of competitively binding to metal ions, such as zinc and calcium, which are essential for proteases such as MMPs, thereby inhibiting the catalytic activity of these proteases. ${ }^{6}$ For PA, three other mechanisms of action have been proposed, ${ }^{28-30}$ in addition to its metal chelating activity similar to CHX, although PA-protease interactions have not yet been well elucidated. First, the protease resistance may be achieved via irreversible conformational changes of proteases within the catalytic domain or allosteric inhibition of other modular domains that coparticipate in collagen biodegradation. ${ }^{2,31}$ Second, PA may indirectly interfere with protease production and activation by modulating host immune responses. ${ }^{32}$ Third, the induction of exogenous crosslinks in dentin matrix by crosslinkers, such as PA, increases the density of the collagen network and reduces the swelling ratio of demineralized dentin, indicating a decrease in the collagenase absorption, ${ }^{33}$ thereby enhancing the matrix resistance against enzymatic degradation. The advantage of inactivating proteolytic enzymes in the dentin matrix by crosslinking is that the mechanism is nonspecific - that is, it crosslinks all types of MMPs and cysteine cathepsins. ${ }^{34}$ These crosslinks involve covalent bonds that are stable over time, unlike the reversible electrostatic binding of CHX..$^{5}$ Accordingly, Macedo et al. ${ }^{35}$ proposed that collagenase inactivation by crosslinking agents should be long-lasting and may be even more effective than using inhibitors because collagenases do not turnover in dentin. Our results for the anti-proteolytic effect of $\mathrm{PA}$, in comparison to $\mathrm{CHX}$, also support this notion.

In accordance with previous reports, ${ }^{16,33}$ PA-biomodified groups challenged with collagenase showed a higher percentage of mixed failures, mainly at the top of the hybrid layer. Our results suggested that the poorly infiltrated demineralized dentin at the bottom of the hybrid layer was mechanically strengthened by PA biomodification and contributed to the stabilisation of the bonding interface.

Clinically, our PA biomodification protocol is less favourable than directively adding PA into adhesive system, because it adds an extra step to the bonding protocol, which is in contrast to the clinician's preference for simplification. However, this may result in a lower risk of interfering with the well-balanced monomer-solvent cocktails. Our previous investigation confirmed that the degree of conversion was not significantly affected by this additional PA biomodification step. ${ }^{16}$ A critical problem encountered with PA biomodification when attempting to achieve durable dentin bonding is that the resin-sparse collagen matrices are still retained within the hybrid layer. The present results further revealed that the $\mu$ TBS values of PA-biomodified specimens 
were reduced than that before degradation and that Hyp was still detectable after enzymatic challenge. So the denuded collagen fibrils are structurally unstable and face enduring challenges as long as they lack protection from resin or apatite. Even if these demineralized collagen fibrils can be stiffened 50-fold with crosslinking agents, the resulting modulus of elasticity is still far inferior to that of resininfiltrated dentin. ${ }^{2}$ Despite the limitations of this protocol, PA biomodification is helpful to limit the negative influence of host-derived proteases on the hybrid layer formed during dentin bonding, to maintain the stability of the bonding interface and to gain time for the slow process of mineral precipitation in resin-sparse regions in the hybrid layer to seal the denuded collagen fibrils (i.e., for remineralisation). ${ }^{36-37}$ In this regard, the application of PA in the bonding procedure could be a beneficial complementary approach to other strategies in the attempt to overcome the critical barriers currently encountered in dentin bonding.

\section{CONCLUSIONS}

Within the limits of this study, it may be concluded that transient PA biomodification of demineralized dentin decreased proteolytic activity on collagen matrices and enhanced the resistance of the bonding interface to enzymatic degradation. This new protocol has the potential to improve bonding durability and could be an adjunct approach to other strategies, such as remineralisation, in an attempt to achieve more durable dentin bonding.

\section{ACKNOWLEDGEMENTS}

The present study was supported by research funds from the Natural Science Foundation of China (No. 81130078 and No. 81000458) and Program for Changjiang Scholars and Innovative Research Team in University (No. IRT13051).

1 Breschi L, Mazzoni A, Ruggeri A et al. Dental adhesion review: aging and stability of the bonded interface. Dent Mater 2008; 24(1): 90-101.

2 Liu Y, Tjaderhane L, Breschi L et al. Limitations in bonding to dentin and experimental strategies to prevent bond degradation. J Dent Res 2011; 90(8): 953-968.

3 Hashimoto $\mathrm{M}$, Ohno $\mathrm{H}$, Kaga $\mathrm{M}$ et al. In vivo degradation of resin-dentin bonds in humans over 1 to 3 years. J Dent Res 2000; 79(6): 1385-1391.

4 Breschi L, Martin P, Mazzoni A et al. Use of a specific MMP-inhibitor (galardin) for preservation of hybrid layer. Dent Mater 2010; 26(6): 571-578.

5 Breschi L, Mazzoni A, Nato F et al. Chlorhexidine stabilizes the adhesive interface: a 2-year in vitro study. Dent Mater 2010; 26(4): 320-325.

6 de Munck J, van den Steen PE, Mine A et al. Inhibition of enzymatic degradation of adhesive-dentin interfaces. J Dent Res 2009; 88(12): 1101-1106.

7 Ricci HA, Sanabe ME, de Souza Costa CA et al. Chlorhexidine increases the longevity of in vivo resin-dentin bonds. Eur J Oral Sci 2010; 118(4): 411-416.

8 Sadek FT, Braga RR, Muench A et al. Ethanol wet-bonding challenges current antidegradation strategy. J Dent Res 2010; 89(12): 1499-1504.

9 La VD, Howell AB, Grenier D. Cranberry proanthocyanidins inhibit MMP production and activity. J Dent Res 2009; 88(7): 627-632.

10 Epasinghe DJ, Yiu CK, Burrow MF et al. The inhibitory effect of proanthocyanidin on soluble and collagen-bound proteases. J Dent 2013; 41(9): 832-839.

11 Castellan CS, Pereira PN, Grande RH et al. Mechnical characterization of proanthocyandin-dentin matrix interaction. Dent Mater 2010; 26(10): 968-973.

12 Castellan CS, Bedran-Russo AK, Karol S et al. Long-term stability of dentin matrix following treatment with various natural collagen cross-linkers. J Mech Behav Biomed Mater 2011; 4(7): 1343-1350.
13 Castellan CS, Bedran-Russo AK, Antunes A et al. Effect of dentin biomodification using naturally derived collagen cross-linkers: one-year bond strength study. Int J Dent 2013; 2013: 918010.

14 Hechler B, Yao X, Wang Y. Proanthocyanidins alter adhesive/dentin bonding strengths when included in a bonding system. Am J Dent 2012; 25(5): 276-280.

15 Liu R, Fang M, Xiao Y et al. The effect of transient proanthocyanidins preconditioning on the cross-linking and mechanical properties of demineralized dentin. J Mater Sci Mater Med 2011; 22(11): 2403-2411.

16 Fang M, Liu R, Xiao Y et al. Biomodification to dentin by a natural crosslinker improved the resin-dentin bonds. J Dent 2012; 40(6): 458-466.

17 Liu RR, Fang M, Zhao SJ et al. [The potential effect of proanthocyanidins on the stability of resin-dentin bonds against thermal cycling.] Zhonghua Kou Qiang Yi Xue Za Zhi 2012; 47(5): 268-272. Chinese.

18 Liu Y, Chen M, Yao X et al. Enhancement in dentin collagen's biological stability after proanthocyanidins treatment in clinically relevant time periods. Dent Mater 2013; 29(4): 485-492.

19 Yamauchi M, Shiiba M. Lysine hydroxylation and cross-linking of collagen. Methods Mol Biol 2008; 446: 95-108.

20 Tezvergil-Mutluay A, Agee KA, Hoshika T et al. The inhibitory effect of polyvinylphosphonic acid on functional matrix metalloproteinase activities in human demineralized dentin. Acta Biomater 2010; 6(10): 4136-4142.

21 Mazzoni A, Mannello F, Tay FR et al. Zymographic analysis and characterization of MMP-2 and -9 forms in human sound dentin. J Dent Res 2007; 86(5): 436-440.

22 Hosaka K, Nishitani Y, Tagami J et al. Durability of resin-dentin bonds to water- vs. ethanol-saturated dentin. J Dent Res 2009; 88(2): 146-151.

23 Sulkala M, Tervahartiala T, Sorsa T et al. Matrix metalloproteinase-8 (MMP-8) is the major collagenase in human dentin. Arch Oral Biol 2007; 52(2): 121-127.

24 Perumal S, Antipova O, Orgel JP. Collagen fibril architecture, domain organization, and triple-helical conformation govern its proteolysis. Proc Natl Acad Sci U S A 2008; 105(8): 2824-2829.

25 Tjaderhane L, Nascimento FD, Breschi L et al. Optimizing dentin bond durability: control of collagen degradation by matrix metalloproteinases and cysteine cathepsins. Dent Mater 2013; 29(1): 116-135.

26 Niu LN, Zhang L, Jiao K et al. Localization of MMP-2, MMP-9, TIMP-1, and TIMP-2 in human coronal dentine. J Dent 2011; 39(8): 536-542.

27 Carrilho MRO, Geraldeli S, Tay F et al. In vivo preservation of the hybrid layer by chlorhexidine. J Dent Res 2007; 86(6): 529-533.

28 La VD, Bergeron C, Gafner S et al. Grape seed extract suppresses lipopolysaccharideinduced matrix metalloproteinase (MMP) secretion by macrophages and inhibits human MMP-1 and -9 activities. J Periodontol 2009; 80(11): 1875-1882.

29 Ebrahimi A, Schluesener H. Natural polyphenols against neurodegenerative disorders: potentials and pitfalls. Ageing Res Rev 2012; 11(2): 329-345.

30 Ku CS, Sathishkumar M, Mun SP. Binding affinity of proanthocyanidin from waste Pinus radiata bark onto proline-rich bovine achilles tendon collagen type I. Chemosphere 2007; 67(8): 1618-1627.

31 Sela-Passwell N, Rosenblum G, Shoham T et al. Structural and functional bases for allosteric control of MMP activities: can it pave the path for selective inhibition? Biochim Biophys Acta 2010; 1803(1): 29-38.

32 Feghali K, Feldman M, La VD et al. Cranberry proanthocyanidins: natural weapons against periodontal diseases. J Agric Food Chem 2012; 60(23): 57285735.

33 Nam K, Kimura T, Kishida A. Physical and biological properties of collagenphospholipid polymer hybrid gels. Biomaterials 2007; 28(20): 3153-3162.

34 Cova A, Breschi L, Nato F et al. Effect of UVA-activated riboflavin on dentin bonding. J Dent Res 2011; 90(12): 1439-1445.

35 Macedo GV, Yamauchi M, Bedran-Russo AK. Effects of chemical cross-linkers on caries-affected dentin bonding. J Dent Res 2009; 88(12): 1096-1100.

36 Tay FR, Pashley DH. Biomimetic remineralization of resin-bonded acid-etched dentin. J Dent Res 2009; 88(8): 719-724.

37 Tay FR, Pashley DH. Guided tissue remineralisation of partially demineralised human dentine. Biomaterials 2008; 29(8): 1127-1137.

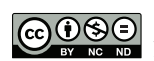

This work is licensed under a Creative Commons Attribution-

NonCommercial-NoDerivs 3.0 Unported License. The images or other third party material in this article are included in the article's Creative Commons license, unless indicated otherwise in the credit line; if the material is not included under the Creative Commons license, users will need to obtain permission from the license holder to reproduce the material. To view a copy of this license, visit http://creativecommons.org/licenses/ by-nc-nd/3.0/ 